\title{
Análisis del disfrute con la educación física, la importancia de las clases y las intenciones de práctica en estudiantes de secundaria: comparativa por género y curso \\ Analysis of the enjoyment of physical education, the importance of classes and the intention of exercising in secondary school students: gender and grade comparisons \\ Vicente Lluna-Ruíz, Mario Alguacil, María Huertas González-Serrano \\ Universidad Católica de Valencia (España)
}

Resumen. La presente investigación analiza la opinión de los estudiantes acerca del papel que ejercen los distintos agentes sociales (familia, amigos, profesor) en su práctica de actividad física de actividad física, así como su habilidad percibida, para finalmente comprobar si existen diferencias en el disfrute, la importancia percibida de las clases y las intenciones de práctica deportiva en función del género y del curso. El estudio se ha llevado a cabo en los cursos de $3^{\circ}$ y $4^{\circ}$ de la ESO y $1^{\circ}$ de Bachillerato de un colegio privado de Valencia (España), con una muestra compuesta por 165 alumnos. Los datos se obtuvieron a partir de un cuestionario validado formado a partir de la literatura existente y orientado a conocer información sobre aspectos como la frecuencia con que su entorno les habla de actividad física, la practican, la practican con los propios estudiantes o les animan a su práctica, la importancia que el entorno le otorga a la educación física y en qué medida esos agentes sociales son importantes para influir en la práctica de actividad física de los estudiantes. Por último, se analiza la habilidad percibida por los estudiantes, su interés hacia la actividad física, su disfrute con ella y sus intenciones de práctica, para posteriormente analizar comparaciones basadas en el género y en el curso al que pertenecen, comprobando en qué aspectos existen o no diferencias significativas en función de dichas variables.

Palabras clave: Actividad física, Educación, Adolescentes, Entorno Social.

\begin{abstract}
This research analyses students' opinions about the role of different social agents (family, friends, professor) in their practice of physical activity, as well as their perceived ability, enjoyment, and sports intentions. The study was carried out in the 3rd and 4th years of secondary school and 1st year of Bachillerato of a private school in Valencia (Spain), with a sample of 165 students. The data were obtained from a validated questionnaire formed from existing literature and aimed at knowing information on aspects such as the frequency with which their social environment talks to them about physical activity, they practice it, practice it with the students themselves or encourage them to practice it, the importance that the social environment attributes to physical education and to what extent these social agents are important in influencing students' practice of physical activity. Finally, students' perceived ability, their interest in physical activity, their enjoyment of it, and their intentions of practice are analysed, and then comparisons based on gender and the course to which they belong are analysed, checking in which aspects there are -or not- significant differences depending on these variables.
\end{abstract}

Keywords: Physical activity, Education, Adolescents, Social Environment.

\section{Introducción}

El sedentarismo está cada vez más asentado como uno de los factores de riesgo de enfermedades crónicas y obesidad (considerada como una de las epidemias más grandes y que más afectan a la mortalidad en los países desarrollados en el último siglo). El sedentarismo es un término relacionado con la inactividad física. Llevar una vida sedentaria favorece la obesidad, que es uno de los factores de riesgo más importantes en relación a los desórdenes cardiovasculares (Lumish, O'Reilly, \& Reilly, 2020). Actualmente, es un hecho reconocido que la práctica habitual de actividad física incrementa la salud, el bienestar y la calidad de vida. Cuesta creer que, a pesar de ser conocidos los grandes beneficios de la práctica de actividad física, los datos que maneja la OMS (2018) indican que, en los últimos estudios globales realizados en el año 2010, el 81\% de los adolescentes entre 11 y 17 años, no se mantienen físicamente activos, siendo más inactivas las chicas (84\%) que los chicos (78\%). Ante estos datos, se hace necesario concienciar a los jóvenes de las consecuencias del sedentarismo y la obesidad (Quiroga et al., 2019).

Fecha recepción: 09-12-19. Fecha de aceptación: 01-05-20

Mario Alguacil Jiménez

mario.alguacil@ucv.es
De manera general, los niños y los jóvenes son más activos que los adultos, pero sus prácticas deportivas van disminuyendo a medida que se van haciendo mayores. De hecho, los estudiantes de la Educación Secundaria Obligatoria (ESO), practican menos que los de Primaria (Castillo, Tornero y García, 2018), siendo por este motivo la adolescencia un periodo que presenta tasas de sedentarismo preocupantes. La inactividad física afecta a los menores, por este motivo se debe crear estrategias y estudiar quién influye y de qué manera a los menores para practicar o no actividad física.

En relación a los beneficios de la educación física, en la última década se han realizado múltiples investigaciones en las que los beneficios de la actividad física se han visto respaldados (Adarve et al., 2019; Estrada et al., 2016). A pesar de esto, sigue siendo importante analizar la actividad física como un factor preventivo, que debe de tener unas pautas individualizadas para cada persona, programando correctamente el ejercicio físico para la salud, y haciendo hincapié en sus valores educativos, sociales y sanitarios, promoviendo además un estilo de vida sano (Casimiro \& Pierón, 2001). Organismos internacionales como la Organización Mundial de la Salud han determinado que como mínimo son necesarios 60 minutos diarios de actividad física de intensidad moderada o incluso vigorosa en personas de entre 5 a 17 años.

En este sentido, para algunos autores (Flores-Paredes, 
Pino-Vanegas, \& Villamar-Barriga, 2020; Ros-Fuentes, 2007), las actividades aeróbicas de baja o moderada intensidad generan unos beneficios específicos no solo a nivel cardiovascular, sino que también se producen mejoras en aspectos como la función mental, la percepción de la imagen corporal y la sensación de bienestar.. Además, el individuo aprende a regular su activación y evade pensamientos, emociones y conductas negativas, evitando así procesos de ansiedad, estrés o depresión y aumentando la autoestima y la seguridad, además de un mejor control emocional (González, Cayuela, \& López, 2019). Para poder obtener los beneficios comentados, es conveniente realizar una práctica de ejercicio físico atractiva, divertida y estimulante, para que la participación por parte de los escolares sea más alta y se consiga una continuidad en la actividad (Gálvez, Rodríguez, \& Velandrino, 2009) ya que se ha demostrado que cuando la diversión es mayor, los participantes otorgan más importancia a la educación física y muestran mayores intenciones de práctica deportiva (González, Gómez-López, \& Granero-Gallegos, 2019).

Por otro lado, en cuanto al papel de la familia en esos hábitos saludables, la influencia de esta fue definida por Pate et al. (2002) y Ornelas, Perreira, \& Ayala (2007) como uno de los principales mecanismos de influencia de la praìctica de la actividad fiìsica por parte de los niños y de los adolescentes, pese a que cada familia puede tener mecanismos diferentes de influencia sobre los haibitos de actividad fiìsica (Soubhi, Potvin, \& Paradis, 2004). Concretamente, los padres ejercen una influencia fundamental en la práctica de actividad física y deporte en los niños y adolescentes (Pelegrín, García \& De los Fayos, 2019). De hecho, el contacto entre padres e hijos facilita la influencia parental en un vasto conjunto de comportamientos, entre el que se encuentra la realizacioìn de actividad fiìsica, ya que ademaìs, la familia puede ser vista como un factor que refleja las influencias culturales, econoìmicas y especiìficas de cada sociedad (Telama, Leskinen, \& Yang, 1996). Por esto, los padres y madres se pueden considerar como los agentes socializadores maìs importantes a la hora de estimular la participacioìn de los hijos en la actividad física (Hohepa et al., 2007; Mota \& Queiros, 1996; Mota \& Sallis, 2002; MuñozGaliano, Hernández-García, \& Torres-Luque, 2019).

La familia, es considerado el primer y más potente agente socializador en las primeras edades, transmisor de valores, comportamientos y normas (Casimiro \& Pierón, 2001; Jaramillo et al., 2017). Por su parte, Berger \& Luckman (1995) en su modelo sobre socialización primaria, explican la importancia que tiene el ámbito familiar como principal agente de socialización. Según este modelo, la socialización como proceso está latente a través de las relaciones y se manifiesta implícitamente mediante la observación, el modelado y el ensayo y error. Además de esto, en las etapas de Primaria y Secundaria, dichos agentes sociales y las características individuales del niño como la autoestima y la motivación propia, son determinantes en el comportamiento (Franco, Coterón, \& Gómez, 2017; McPherson, Curtis, \& Loy, 1989).

La sociedad influye en la familia, y muchos de los recursos educativos que utiliza dicha familia, vienen marcados por la sociedad a la que pertenecen (Rodríguez, 2004). Debemos tener en cuenta también que los niños tienden a la imita- ción, mientras que los adolescentes ejecutan sus comportamientos sociales utilizando la comunicación interpersonal (Torre, 1998) es por ello que, en las etapas más tempranas, las conductas del entorno, en este caso de la familia, pueden ser más influyentes en el comportamiento de los escolares y en el establecimiento de pautas y hábitos saludables que en etapas posteriores.

Además, continuando con la información relativa al entorno, vemos que las relaciones sociales de los adolescentes pueden facilitar conductas que motiven hacia la actividad física, aunque también pueden llegar a dificultarlas (Lizandra y Peiró-Velert, 2020). Por lo tanto, el comportamiento que tienen los padres puede favorecer o perjudicar la aparición de actitudes positivas de la personalidad durante la ejecución deportiva (Sánchez, 1997). En este sentido, la actitud positiva de los padres y madres dirigida hacia la actividad físico-deportiva extraescolar de sus hijos influye en el interés de éstos por estas prácticas (Ruiz-Juan, 2000). Es por ello que los componentes familiares deben crear un clima positivo para la práctica de actividades físico-deportivas, deben aumentar y promover los momentos para practicar deporte y tienen que actuar como modelos (Nuviala, 2003) ya que los padres ejercen influencia en la creación de modelos de vida saludables y la creación de conductas (Cordente, García, \& Sillero, 2008). Por otro lado, el nivel de práctica y las valoraciones de los padres, así como el nivel de educación, la existencia de figuras deportistas dentro de la familia o la actitud hacia la práctica deportiva, ejercen una influencia positiva en la participación de los niños en las actividades deportivas (Kotan, Hergüner, \& Yaman, 2009). Otros aspectos del entorno, como la clase social de los padres, también es un elemento influyente en la práctica deportiva, existiendo un mayor desinterés hacia el deporte en las familias que pertenecen a clases sociales más bajas (Campos-Granell, 2011).

Por todo ello, entre los objetivos del presente estudio encontramos, en primer lugar, la intención de conocer a nivel descriptivo la distribución de opiniones de los estudiantes respecto a su práctica de actividad física y la de su entorno, así como la importancia que este entorno tiene para ellos a la hora de practicar actividad física y qué importancia le concede a la práctica deportiva. En segundo lugar, se pretende analizar el disfrute con la educación física, la importancia que otorgan a las clases y las intenciones futuras de práctica de los estudiantes, comparando dichos resultados en función del género y el curso para determinar si existen o no diferencias significativas en este sentido ente los grupos analizados.

\section{Método}

\section{Participantes}

Esta investigación se desarrolló en un colegio privado de la ciudad de Valencia (España), con un total de 165 participantes pertenecientes a los cursos de $3^{\circ}$ de la ESO, $4^{\circ}$ de la ESO y $1^{\circ}$ de Bachillerato (edades comprendidas entre los 14 y los 16 años). Del total de la muestra de estudiantes, 81 de ellos son hombres (49.09\%) y 84 mujeres (50.91\%). Respecto al curso, vemos que en tercer curso hay 55 estudiantes (33.33\%), en $4^{\circ}$ hay $48(29.09 \%)$ y en $1^{\circ}$ de bachillerato hay un total de $62(37.58 \%)$. Los criterios de selección de la muestra 
han sido ser estudiantes del centro y haber cumplimentado y firmado adecuadamente el consentimiento informado que se les distribuyó a los alumnos de forma previa al estudio.

\section{Instrumento}

Para la confección del instrumento mediante el cual poder recoger la información necesaria para el estudio, se ha utilizado el cuestionario validado por Aznar (1998) y posteriormente utilizado por Montil (2004). El instrumento, en línea con el objetivo del estudio, está orientado a recoger información sobre la percepción de la autoeficacia, la actitud hacia la actividad física y la educación física, las actitudes y conductas de los miembros de su entorno, la adherencia a la actividad física, las intenciones de práctica en el futuro, así como los aspectos relacionados con la salud.

El instrumento utilizado consta de 23 preguntas, además de la recogida de aspectos como el curso al que pertenecían y el género. Detallando el instrumento forma más concreta, encontramos un primer bloque (preguntas de la 1 a la 8) donde se habla acerca de la unidad familiar, en la que se atiende al nivel socio cultural y económico de los padres y madres y a su posible asociación a algún tipo de club o federación. En el segundo bloque (preguntas de la 9 a la 14) se pregunta sobre la influencia del modelo, el apoyo social y la influencia social de los «otros significativos» propuestos como determinantes de la conducta de Actividad Física desde el modelo Socio-cognitivo de Taylor et al. (1994), adaptadas por el cuestionario que comentamos de Aznar (1998). Para la medición del grado en que los sujetos perciben las diferentes actitudes y conductas se utilizó una escala de respuesta tipo Likert que iba desde «nunca» a «siempre». Posteriormente, la pregunta número 15 busca recoger información sobre la autoeficacia percibida en relación con la habilidad del estudiante frente a los demás, donde los encuestados podían elegir responder que eran más hábiles que sus compañeros, igual de habilidosos o menos habilidosos que los demás, categorizando esa percepción. Encontramos un tercer bloque (preguntas 16 a 22), que utilizan escala de respuesta tipo Likert, con un rango que varía desde «no, es muy desagradable» hasta «si, es muy agradable» en algunos enunciados y desde «no, es muy poco probable» hasta «si, es probable» en otros. La intención de este bloque 3 es recoger información sobre el componente afectivo de las actitudes hacia la Actividad Física y la posibilidad de realizar actividad física en un futuro. Por último, la pregunta 23 consta de un enunciado sobre su práctica de actividad física en el que los alumnos debían responder eligiendo la afirmación que más se ajustara a su realidad.

Finalmente, en cuanto a las propiedades psicométricas del cuestionario, cabe decir que dicho instrumento ha sido validado previamente para el mismo contexto por los autores referenciados y que la fiabilidad de las escalas en este estudio ha sido de .85, de la misma forma que, en otros ejemplos, como el estudio de Pantoja-Vallejo y Montijano-González (2012) también obtuvo una fiabilidad adecuada (á= .93) superando el criterio de .70 establecido por la literatura (Hair et al., 2006).

\section{Procedimiento}

Se desarrolloì un protocolo de actuación sobre lo que se realizaría durante todo el proceso, tanto antes como durante y después del momento de presentar los cuestionarios a los alumnos. Siguiendo los principios éticos y códigos de conducta de la American Psychological Association (2002), se preparó un consentimiento informado para que fuese autorizado por los padres y/o tutores de los estudiantes, explicando los objetivos que se perseguían en el estudio, además se mantuvo en todo momento el anonimato y confidencialidad de la información recogida y no hubo ningún tipo de discriminación en la selección de la muestra, únicamente el hecho de no pertenecer a los cursos que se pretendían analizar. El centro educativo se encargó de repartir las autorizaciones a los alumnos y de poner una fecha límite de entrega. Posteriormente, se elaboró una lista con los alumnos participantes por cada clase y el profesor de Educación Física proporcionó un horario de sus clases para organizar la recogida de datos de forma más efectiva y eficiente. Finalmente, se concertó el día y las horas en la que se realizarían los cuestionarios y se recogieron las encuestas.

\section{Análisis estadístico}

El análisis estadístico de los datos se llevó a cabo mediante el paquete estadístico SPSS 25. En primer lugar, se realizó un análisis de fiabilidad de las escalas para comprobar que el valor de alfa de Cronbach era adecuado y que, por tanto, las escalas utilizadas eran fiables. Posteriormente, se extrajeron los datos para realizar un análisis descriptivo de las preguntas relacionadas con la práctica deportiva, la habilidad percibida, así como la práctica deportiva del entorno y la importancia que le conceden a la actividad física. Finalmente, con este mismo software se llevaron a cabo comparaciones en función del género, mediante la realización de la prueba $\mathrm{T}$ para muestras independientes, y en función del curso al que pertenecen los estudiantes, en este caso mediante la realización de la prueba ANOVA.

\section{Resultados}

\section{Resultados descriptivos}

En primer lugar, encontramos un bloque en el que las preguntas van orientadas a conocer la frecuencia con que su entorno les habla de actividad física, la practican, la practican con los propios estudiantes o les animan a su práctica. En la primera pregunta «¿Con qué frecuencia cada una de estas personas te habla acerca de la actividad física?», como se puede apreciar en la tabla 1 , los resultados más destacados indican que el $29,1 \%$ de los encuestados declara que sus amigos les hablan a menudo a cerca de Actividad Física y un 33,3\% que su profesor de Educación Física les habla siempre sobre Actividad Física, lo que resulta lógico.

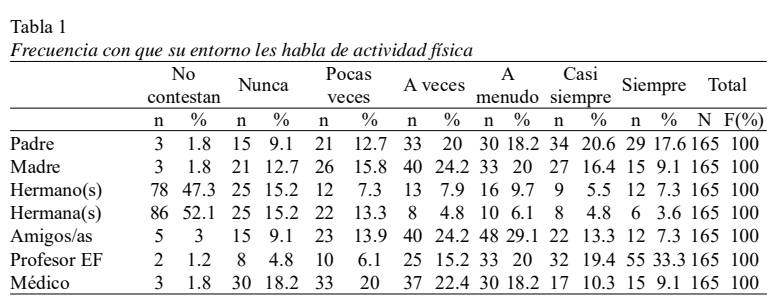

Siguiendo con esta línea, pero en este caso orientada a la pregunta «¿Con qué frecuencia cada una de estas personas 
hace Actividad Física?» (ver tabla 2) el 30,3\% de los alumnos señala que su madre hace pocas veces actividad física, el 48,5\% dice que su profesor de Educación Física siempre hace Actividad Física, siendo un porcentaje que habría que analizar, para ver si tienen en cuenta el horario lectivo o actividades que observan de él fuera del ámbito escolar, mientras que el $29,7 \%$ apunta que sus amigos a veces realizan actividad física.

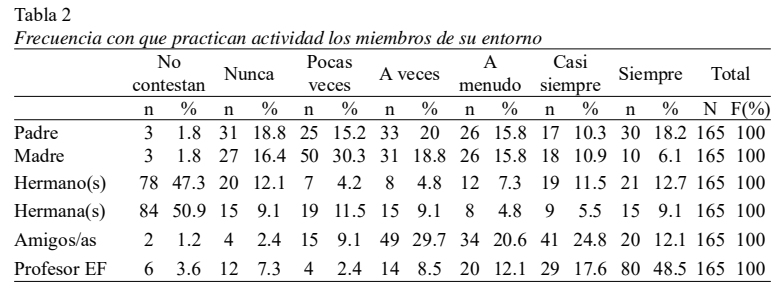

Respecto a la pregunta «¿Con qué frecuencia cada una de estas personas hace Actividad Física contigo?», cuyas respuestas aparecen en la tabla 3 , podemos ver, por ejemplo, como el 54,5\% de los encuestados nunca realiza actividad física con su madre y el $43 \%$ nunca realiza actividad física con su padre. Por otro lado, solo el $1,2 \%$ de la muestra dice que siempre practica actividad física con su hermano(s) o hermana(s) y un $29,7 \%$ dice practicar a veces actividad física con los amigos y amigas.

Tabla 3

\begin{tabular}{|c|c|c|c|c|c|c|c|c|c|c|c|c|c|c|}
\hline & \multicolumn{2}{|c|}{$\begin{array}{c}\text { No } \\
\text { contestan }\end{array}$} & \multicolumn{2}{|c|}{ Nunca } & \multicolumn{2}{|c|}{$\begin{array}{l}\text { Pocas } \\
\text { veces }\end{array}$} & \multicolumn{2}{|c|}{ A veces } & \multicolumn{2}{|c|}{$\begin{array}{c}\text { A } \\
\text { menudo }\end{array}$} & \multicolumn{2}{|c|}{$\begin{array}{c}\text { Casi } \\
\text { siempre }\end{array}$} & \multicolumn{2}{|c|}{ Siempre Total } \\
\hline & $\mathrm{n}$ & $\%$ & $\mathrm{n}$ & $\%$ & $\mathrm{n}$ & $\%$ & $\mathrm{n}$ & $\%$ & $\mathrm{n}$ & $\%$ & $\mathrm{n}$ & $\%$ & $\begin{array}{llll}\mathrm{n} & \% & \mathrm{~N} \\
\end{array}$ & N F $(\%)$ \\
\hline adre & 3 & 1.8 & 71 & 43 & 35 & 21.2 & 34 & 20.6 & 9 & 5.5 & 6 & 3.6 & $\begin{array}{lll}7 & 4.2 & 16\end{array}$ & $165 \quad 100$ \\
\hline Madre & 3 & 1.8 & 90 & 54.5 & 38 & 23 & 16 & 9.7 & 13 & 7.9 & 2 & 1.2 & $\begin{array}{lll}3 & 1.8 & 16\end{array}$ & 165100 \\
\hline Hermano(s) & 79 & 47.9 & 46 & 27.9 & 8 & 4.8 & 15 & 9.1 & 10 & 6.1 & 5 & 3 & $\begin{array}{lll}2 & 1.2 & 16\end{array}$ & 165100 \\
\hline Hermana(s) & 79 & 47.9 & 47 & 28.5 & 15 & 9.1 & 13 & 7.9 & 6 & 3.6 & 3 & 1.8 & $\begin{array}{lll}2 & 1.2 & 16\end{array}$ & 165100 \\
\hline Amigos/as & 0 & 0 & 25 & 15.2 & 29 & 17.6 & 49 & 29.7 & 25 & 15.2 & 19 & 11.5 & $18 \quad 10.916$ & 165100 \\
\hline Profesor EF & 1 & .6 & 65 & 39.4 & 20 & 12.1 & 27 & 16.4 & 10 & 6.1 & 15 & 9.1 & $27 \quad 16.416$ & $165 \quad 100$ \\
\hline
\end{tabular}

En la siguiente pregunta, para concluir con este primer bloque, se intenta conocer en qué medida el entorno del estudiante le anima a la práctica de actividad física. Para ello, a través de la pregunta «¿Con qué frecuencia cada una de estas personas te anima para que hagas Actividad Física?» (ver tabla 4) el 40,6\% de los alumnos contesta que su profesor de educación física siempre le anima a practicar actividad física. Por otro lado, también vemos que el $32,7 \%$ apunta que su padre siempre le anima y el $28,5 \%$ que declara lo mismo por parte de su madre. En este sentido, el 9,7\% de la muestra dice que su padre y su madre nunca le animan a hacer actividad física. Finalmente, el 18,8\% indica que los amigos a veces les animan a la práctica.

\begin{tabular}{|c|c|c|c|c|c|c|c|c|c|c|c|c|c|c|c|c|}
\hline & \multicolumn{2}{|c|}{$\begin{array}{c}\text { No } \\
\text { contestan }\end{array}$} & \multicolumn{2}{|c|}{ Nunca } & \multicolumn{2}{|c|}{$\begin{array}{l}\text { Pocas } \\
\text { veces }\end{array}$} & \multicolumn{2}{|c|}{ A veces } & \multicolumn{2}{|c|}{$\begin{array}{c}\mathrm{A} \\
\text { menudo }\end{array}$} & \multicolumn{2}{|c|}{$\begin{array}{c}\text { Casi } \\
\text { siempre }\end{array}$} & \multicolumn{2}{|c|}{ Siempre } & \multicolumn{2}{|c|}{ Total } \\
\hline & $\mathrm{n}$ & $\%$ & $\mathrm{n}$ & $\%$ & $\mathrm{n}$ & $\%$ & $\mathrm{n}$ & $\%$ & $\mathrm{n}$ & $\%$ & $\mathrm{n}$ & $\%$ & $\mathrm{n}$ & $\%$ & & $\mathrm{F( \% )}$ \\
\hline & 3 & 1.8 & 16 & 9.7 & 11 & 6.7 & 181 & 10.9 & 29 & 17.6 & 34 & 20.6 & 54 & 32.7 & 1651 & 100 \\
\hline & 3 & 1.8 & 16 & 9.7 & 12 & 7.3 & 28 & 17 & 28 & 17 & 31 & 18.8 & 47 & 28.5 & 1651 & 100 \\
\hline Hermano & 82 & 49.7 & 301 & 18.2 & 7 & 4.2 & 12 & 7.3 & 11 & 6.7 & 7 & 4.2 & 16 & 9.7 & 1651 & 100 \\
\hline rmal & 81 & 49.1 & 321 & 19.4 & 12 & 7.3 & 15 & 9.1 & 9 & 5.5 & 3 & 1.8 & 13 & 7.9 & 1651 & 100 \\
\hline Amigos/as & 1 & 6 & 261 & 15.8 & 30 & 18.2 & 311 & 18.8 & 24 & 14.5 & 23 & 13.9 & 30 & 18.2 & 1651 & 100 \\
\hline Profesor EF & 2 & 1.2 & 171 & 10.3 & 9 & 5.5 & 301 & 18.2 & 15 & 9.1 & 25 & 15.2 & 67 & 40.6 & 1651 & 100 \\
\hline
\end{tabular}

El segundo bloque va orientado a conocer la importancia que le da el entorno de los estudiantes a la actividad física y el deporte, así como en qué medida son importantes los distintos miembros de su entorno para ayudarle a practicar actividad física. En el primer enunciado de este bloque «Valora la importancia que le dan a la actividad física y al deporte cada una de estas personas», como se puede apreciar en la tabla 5 , un $35,8 \%$ de los padres dan mucha importancia a la actividad física, por un $27,5 \%$ de las madres. Cabe destacar que el 3\% de los alumnos afirman que el profesor de educación física no da nada de importancia a la actividad física y al deporte y que de los amigos un $24,8 \%$ considera que es bastante importante y un $18,8 \%$ que es muy importante, por lo que más de la mitad de los amigos no considera a ese nivel de importancia la educación física.

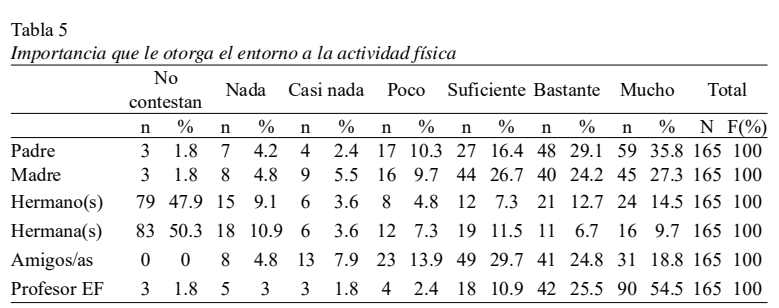

En la segunda pregunta de este bloque sobre la importancia de los miembros del entorno, a la pregunta « $i$ Son estas personas importantes para ti a la hora de ayudarte a participar en los deportes y actividad física?» (ver tabla 6) podemos ver que el $36,4 \%$ de los encuestados cree que su padre es muy importante a la hora de ayudarle a practicar deporte, por un $30,9 \%$ que opina lo mismo de su madre. Por otro lado, el $23,6 \%$ de la muestra piensa que la ayuda que recibe por parte de sus amigos para practicar actividad física y deporte es suficiente, mientras que un $23,6 \%$ indica que su profesor es muy importante para este fin.

\begin{tabular}{|c|c|c|c|c|c|c|c|c|c|c|c|c|c|}
\hline & No & ontestan & $\mathrm{Nad}$ & & $\begin{array}{l}\text { Casi } \\
\text { nada }\end{array}$ & Poco & Sufic & ciente B & Bastar & & Mucho & & Total \\
\hline & $\mathrm{n}$ & $\%$ & $\mathrm{n}$ & $\%$ & $n \quad \% \quad r$ & $\begin{array}{ll}\mathrm{n} & \% \\
\end{array}$ & $\mathrm{n}$ & $\% \quad 1$ & $\mathrm{n} \quad 0$ & $\%$ & n $\%$ & $\mathrm{~N}$ & $\mathrm{~F}(\%)$ \\
\hline Padre & 3 & 1.8 & 221 & 13.3 & 95.51 & $\begin{array}{ll}10 & 6.1 \\
\end{array}$ & 26 & 15.83 & $35 \quad 21$ & 1.2 & 6036.4 & 165 & 100 \\
\hline Madre & 3 & 1.8 & 241 & 14.5 & 95.51 & $11 \quad 6.7$ & 30 & 18.23 & $37 \quad 22$ & 2.4 & $\begin{array}{lll}51 & 30.9\end{array}$ & 165 & 100 \\
\hline Hermano(s) & 79 & 47.9 & 221 & 13.3 & $\begin{array}{lll}3 & 1.81\end{array}$ & 148.5 & 16 & 9.71 & 106 & .1 & $\begin{array}{ll}21 & 12.7\end{array}$ & 7165 & 100 \\
\hline Hermana(s) & 81 & 49.1 & 231 & 13.9 & 42.41 & $11 \quad 6.7$ & 16 & 9.71 & 137. & .9 & 1710.3 & 165 & 100 \\
\hline Amigos/as & 0 & 0 & 221 & 13.31 & & $\begin{array}{ll}32 & 19.4\end{array}$ & 39 & 23.63 & $\begin{array}{ll}31 & 18 \\
8\end{array}$ & 8.8 & $\begin{array}{ll}31 & 18.8\end{array}$ & 165 & 100 \\
\hline Profesor EF & 1 & .6 & $21 \quad 1$ & 12.71 & 169.72 & 2313.9 & 32 & 19.43 & 332 & 203 & 3923.6 & 5165 & 100 \\
\hline
\end{tabular}

En el segundo enunciado, se les indica: 'Marca con una ' $\mathrm{X}$ ' la frase que se ajuste más a ti.» (ver tabla 7). El 47,3\% de los encuestados admite hacer ejercicio regularmente, y podemos afirmar que el 95,8\% realiza o estaría interesado en realizar actividad física, frente a un 3,6\% que ni realiza ni les interesa.

\begin{tabular}{lcc} 
Tabla 7 & & \\
Nivel de práctica y de interés hacia la actividad física & $\mathrm{n}$ & $\%$ \\
\hline & 1 & .6 \\
\hline No contestan & 6 & 3.6 \\
No hago ejercicio y no tengo interés & 29 & 17.6 \\
No hago ejercicio, pero quiero hacer & 39 & 23.6 \\
Hago ejercicio de vez en cuando & 12 & 7.3 \\
Hago ejercicio regularmente (varias veces por semana) desde hace menos de 6 meses & 78 & 47.3 \\
Hago ejercicio regularmente desde hace más de 6 meses & &
\end{tabular}

\section{Análisis descriptivo del disfrute y las intenciones de práctica deportiva}

Dentro del instrumento utilizado, encontramos siete enunciados orientados a conocer el disfrute y las intenciones de practicar actividad física por parte de los estudiantes. Dichos enunciados, tienen un tipo de respuesta tipo Likert de 1 a 5 puntos, donde 1 significa estar totalmente en desacuerdo con la afirmación y 5 totalmente de acuerdo. Los resultados de este análisis descriptivo (ver tabla 8) indican que la mejor valoración se encuentra en la pregunta ¿Es probable que hagas actividad física y deporte varias veces por sema- 
na cuando seas mayor? (4.82 \pm 1.35$)$, seguida de la pregunta ¿disfrutas con la actividad física y los deportes? $(4.69 \pm 1.32)$. Por otro lado, la peor valoración aparece en la pregunta ¿cuál crees que es tu estado de salud? donde la media es de 3.84 $( \pm .86)$.

\section{Tabla 8}

Descriptivos del disfrute y las intenciones de práctica de actividad física y deporte

\begin{tabular}{lccc}
\hline & $\mathrm{N}$ & Media & $\mathrm{DT}$ \\
\hline 1. ¿Disfrutas con la AF y los deportes? & 165 & 4.69 & 1.32 \\
2. ¿Disfrutas de las clases de EF del colegio? & 165 & 4.36 & 1.27 \\
3. ¿Es probable que hagas AF varias veces por semana el próximo verano? & 165 & 4.46 & 1.68 \\
4. ¿Crees que las clases de EF son importantes para ti? & 165 & 4.21 & 1.49 \\
5. ¿Cuál crees que es el estado de tu salud? & 165 & 3.84 & .86 \\
6. ¿Es probable que hagas AF varias veces por semana fuera de la escuela & 165 & 4.61 & 1.64 \\
el próximo año? & & & \\
7. ¿Es probable que hagas AF y deporte varias veces por semana cuando & & & \\
seas mayor? & 165 & 4.82 & 1.35 \\
\hline
\end{tabular}

Comparaciones del disfrute, la importancia de las clases y las intenciones de práctica de actividad física en función del género

Una vez conocidos los valores descriptivos de los enunciados orientados al disfrute y a la práctica de actividad física, a continuación, se realiza un análisis comparativo en función del género, para conocer cuáles son los resultados de hombres y de mujeres y en qué medida existen o no diferencias en los distintos enunciados. En la tabla 9, se observa que la media de los hombres es siempre superior a la de las mujeres. En cuanto a las diferencias, vemos que dichas diferencias existen en la pregunta 1 acerca del disfrute con la actividad física y el deporte $(\mathrm{p}<.001)$, la pregunta 2 sobre el disfrute con las clases de educación física $(\mathrm{p}<.05)$, la pregunta 3 referida a la probabilidad de practicar actividad física el próximo verano $(\mathrm{p}<.001)$, la pregunta 4 relacionada con la importancia de las clases de educación física $(\mathrm{p}<.05)$, la pregunta 6 que trata de la probabilidad de hacer actividad física fuera del ámbito escolar $(\mathrm{p}<.001) \mathrm{y}$, finalmente, la pregunta 7 que es la relacionada con la intención de realizar actividad física cuando sea mayor $(\mathrm{p}<.05)$.

Tabla 9

Comparativa del disfrute y las intenciones de práctica de actividad física en función del género

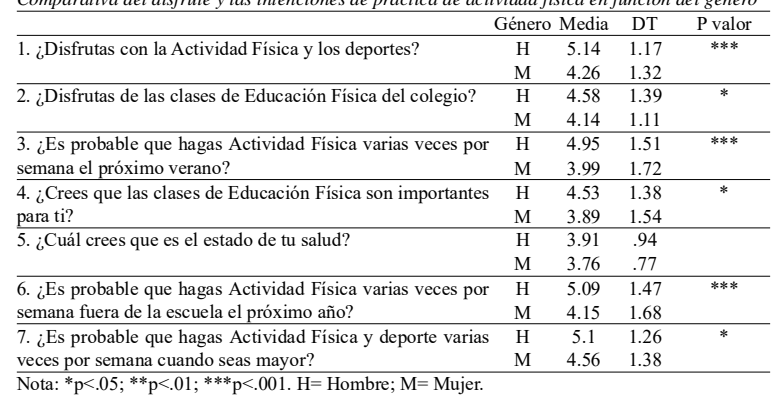

Comparaciones del disfrute, la importancia de las clases y las intenciones de práctica de actividad física en función del curso

Del mismo modo, para conocer los resultados en función del curso, y si existen o no diferencias entre ellos, se ha llevado a cabo un análisis de ANOVA (tabla 10). Los resultados indican que, en la pregunta 2 relacionada con el disfrute de las clases de educación física, existen diferencias significativas entre $3^{\circ} \mathrm{ESO}$ y $4^{\circ} \mathrm{ESO}(\mathrm{p}<.001)$ y entre $3^{\circ} \mathrm{ESO}$ y $1^{\circ}$ de Bachiller $(\mathrm{p}<.05)$. En la pregunta 4 orientada a la importancia de las clases de educación física, podemos ver que hay diferencias significativas entre $3^{\circ} \mathrm{ESO}$ y $4^{\circ} \mathrm{ESO}(\mathrm{p}<.001)$ y entre $3^{\circ} \mathrm{ESO}$ y $1^{\circ}$ de Bachiller $(\mathrm{p}<.01)$. Por otro lado, en la pregunta
6 sobre la probabilidad de hacer actividad física fuera del ámbito escolar, encontramos diferencias significativas entre los cursos de $3^{\circ} \mathrm{ESO}$ y $1^{\circ}$ de Bachiller $(\mathrm{p}<.001)$ así como entre $4^{\circ} \mathrm{ESO}$ y $1^{\circ}$ de Bachiller $(\mathrm{p}<.05)$. Finalmente, en la pregunta 7 acerca de las intenciones de hacer actividad física cuando sean mayores, podemos ver que hay diferencias significativas entre $3^{\circ}$ ESO y $1^{\circ}$ de Bachiller $(\mathrm{p}<.05)$.

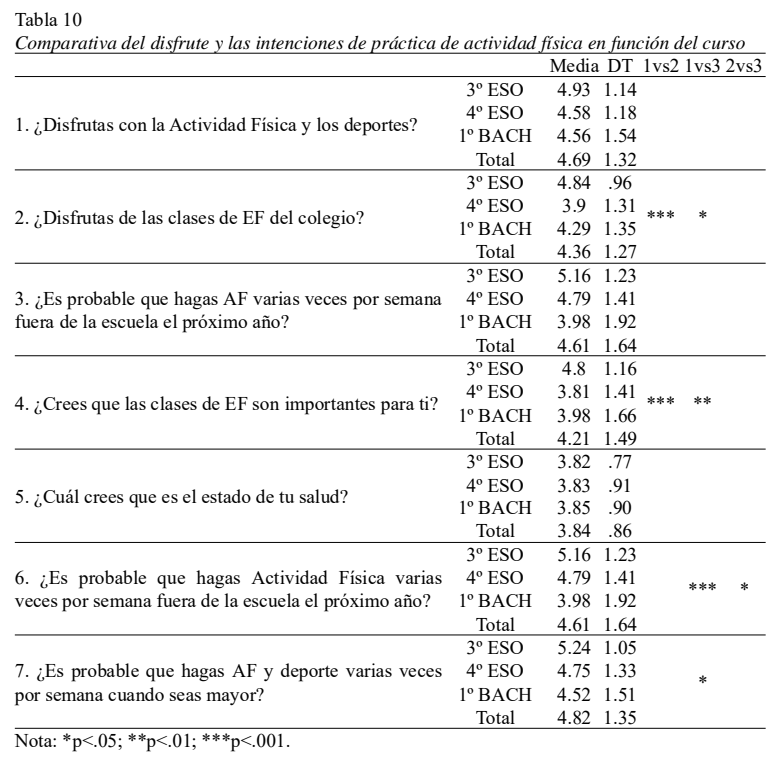

\section{Discusión}

La influencia de la familia es uno de los principales mecanismos de influencia de la praìctica de actividad fì̀sica en niños y adolescentes (Ornelas et al., 2007) pese a que lógicamente cada contexto y cada familia puede tener mecanismos diferentes de influencia sobre los haibitos de actividad fiìsica (Soubhi, Potvin, \& Paradis, 2004). Dentro de los agentes del entorno de los estudiantes, los padres y madres son los más importantes a la hora de estimular la participacioìn de los hijos en la actividad física (Hohepa et al., 2007) especialmente en la población joven. Teniendo en cuenta el valor de las expectativas (Eccles \& Harold, 1991) las percepciones de los padres sobre las habilidades de los hijos son un fuerte predictor de la propia autoeficacia, es por ello que su figura es tan importante. Además de esto, como explicamos en el presente estudio, también los amigos son considerados un gran apoyo, así como resulta importante la figura del profesor de educación física.

Respecto a la edad, los estudiantes del primer ciclo se sienten maìs apoyados por sus padres y madres, seguido de los hermanos y el profesor de educacioìn fiìsica, mientras que, para los alumnos del segundo ciclo, esa influencia cambia y depende más de los hermanos y hermanas que de otros agentes sociales (Leskinen et al., 2000). Esto va en la línea de algunos estudios que indican que la influencia de los padres sobre la práctica de actividad física de los hijos tiende a ser menor a medida que estos crecen (Carratalaì, 1997). Atendiendo a la percepción de autoeficacia de los estudiantes, estudios como el de Bois et al. (2005) comprueban que los apoyos ofrecidos por los padres están relacionados positivamente con la percepción de autoeficacia que tienen los escolares, mientras que los adolescentes señalan a los amigos como uno de los agentes del que más apoyo reciben 
(Van der Horst et al., 2007) aunque también existen otros factores que pueden ser determinantes, como es el disfrute de la actividad o el status socioeconómico (Stucky-Ropp \& DiLorenzo, 1993) o el contexto en el que se desarrollan.

Respecto a las posibles diferencias que surgen del género de los estudiantes, encontramos estudios que dicen que claramente debe haber diferencias entre ellos (Bramham, 2003). Algunas investigaciones proponen que las chicas presentan actitudes maìs favorables hacia la escuela que los chicos, mientras que los chicos son maìs favorables de cara a las clases de educacioìn fiìsica (Pieìron, Ledent, Almond, Airstone, \& Newberry, 1996). Por otro lado, estudios como el de Beltrán et al. (2017) muestran que existen diferencias en función del género en relación al tiempo de actividad física y sedentaria que realizan, donde las chicas muestran niveles más bajos. Estos datos se reflejan también en el estudio de (López, 2019) donde se indica que el 60\% de los chicos y el $82 \%$ de las chicas no siguen las recomendaciones de actividad física, aludiendo a la necesidad de crear programas de actividad física en los que coordine la labor docente con el entorno de los adolescentes, especialmente en el caso de las chicas, a las que se les tiene que hacer una propuesta que les haga ver la actividad física de forma más atractiva. Esta situación se refleja también en el contexto educativo de otros países, donde las chicas perciben más barreras para la práctica de actividad física, reforzando la idea de que en las intervenciones que se planteen la variable género debe tenerse muy en cuenta (Blanco-Ornelas et al., 2019).En relación a la práctica deportiva del entorno del estudiante, encontraron que solo el $22,8 \%$ de los padres y el $20,3 \%$ de las madres realizan actividad fiìsica dos oì maìs días a la semana, mientras que como se ha podido comprobar en este estudio, los valores actuales son superiores, fruto de una mayor conciencia y organización del deporte a todos los niveles, lo que unido al fomento del deporte desde las instituciones, contribuye a que cada vez más gente se inicie o mantenga la práctica deportiva.

\section{Conclusiones}

Como conclusiones del estudio, en primer lugar, acerca de quién les habla a los estudiantes sobre actividad física, los amigos y los profesores de educación física son las figuras más señaladas. Por otro lado, respecto a la práctica de actividad física del entorno, los padres y profesores son los que más practican de forma frecuente, seguidos de los hermanos y amigos, mientras que las madres aparecen en último lugar, siendo los profesores y amigos, seguidos de los padres, las figuras que más practican deporte con los encuestados.

En cuanto a quiénes los animan a practicar actividad física, el profesor, los padres y madres, respectivamente, destacan por encima del resto, ocurriendo lo mismo cuando se analiza el aspecto de quién le da más importancia a la práctica de actividad física. En este sentido, en relación a cómo de importantes consideran los estudiantes a las figuras de su entorno, los padres, seguidos de las madres y los profesores, son las figuras más elegidas.

Por otro lado, respecto a la habilidad percibida, la mayoría de los encuestados se considera un poco por encima de sus compañeros, mientras que, en cuanto a los niveles de práctica, la mayoría considera que su práctica ha sido regular desde hace más de 6 meses.

Finalmente, respecto a las comparaciones estadísticas relacionadas con el disfrute y la importancia de las clases y las intenciones de práctica de actividad física en función del género y del curso, podemos decir, en primer lugar, que existen diferencias significativas entre hombres y mujeres tanto en el disfrute como en las intenciones de práctica, con valoraciones medias más altas siempre en el caso de los hombres. Del mismo modo, en segundo lugar, también encontramos diferencias significativas en el disfrute y la importancia de las clases de educación física, así como en las intenciones futuras de práctica de actividad física y deporte entre los cursos analizados, principalmente entre $3^{\circ} \mathrm{ESO}$ y $4^{\circ} \mathrm{ESO}$ y entre $3^{\circ} \mathrm{ESO}$ y $1^{\circ}$ bachillerato, donde las mejores valoraciones aparecen de forma muy mayoritaria en el curso de $3^{\circ}$ ESO.

\section{Limitaciones y líneas futuras de investigación}

En cuanto a las limitaciones del estudio, encontramos en primer lugar la muestra, ya que, aunque 165 participantes puede ser un número adecuado para un estudio como este, en el que se pretenden conocer algunas variables a nivel descriptivo y comparar otras en función del género y el curso, sin duda una muestra más amplia habría permitido obtener una mayor información útil. Por otro lado, solo se ha analizado un centro de carácter privado, por lo que futuras investigaciones debe ir orientadas a incluir en el análisis distintos tipos de centros, para ver si la titularidad de los mismos puede suponer diferencias en algún sentido, así como el nivel socioeconómico de los barrios donde se encuentran lo que permitiría analizar posibles diferencias que vengan determinadas por los distintos perfiles de centro que se pueden encontrar, acotando más el ámbito de actuación y por tanto segmentando el análisis para hacerlo más específico. Finalmente, decir que el instrumento utilizado supone una cierta limitación puesto que, en los datos referentes a la práctica y el papel del entorno cercano de los adolescentes, no se pueden extraer resultados más allá del análisis descriptivo. Aunque este no era el objetivo principal del artículo, ya que se pretendía testar, principalmente el disfrute con la educación física, la importancia de las clases y las intenciones de práctica, donde se han llevado a cabo comparaciones estadísticas. Para propuestas futuras, se debería intentar dar solución a dichas limitaciones, para que se pueda llegar con mayor profundidad a los problemas que se analizan.

\section{Referencias}

Adarve, M. G., Ortega, F. Z., Sánchez, V. G., Ruz, R. P., \& Sánchez, A. J. L. (2019). Influencia de la práctica de actividad física en el autoconcepto de adolescentes. Retos, 36(36), 342-347.

American Psychological Association. (2002). Ethical principles of psychologists and Code of Conduct. American Psychologist, 57(12), 1060-1073

Aznar, S. (1998). Environmental and significant others' influences on children's physical activity behaviour. 
Tesis doctoral. University of Bristol.

Berger, P. \& Luckmann, T. (1995). La construcción social de la realidad. Amorrortu ediciones.

Blanco, J. R., Valenzuela, M. C. S., Benítez-Hernández, Z. P., Fernández, F. M., \& Jurado, P. J. (2019). Barreras para la práctica de ejercicio físico en universitarios mexicanos comparaciones por género. Retos: nuevas tendencias en educación física, deporte y recreación, (36), 80-82.

Bois, J.E., Sarrazin, P.G., Brustad, R.J., Trouilloud, D.O., \& Cury, F. (2005). Elementary schoolchildren's perceived competence and physical and physical activity involvement: the influence of parents' role modeling behaviors and perceptions of their child's competence. Psychology. Sport Exercise. 6, 381-397. https://doi.org/ 10.1016/j.psychsport.2004.03.003

Campos-Granell, J.C. (2011). Análisis de los determinantes sociales que intervienen en el proceso de detección de talentos. Colección ICD: Investigacioìn en Ciencias del Deporte, (3).

Carratalá, V. (1997). La influencia de los agentes sociales en la participación deportiva en la adolescencia. (Tesis doctoral). Universidad de Valencia. Valencia.

Castillo, E., Tornero, I., \& García, J.A. (2018). Relación entre actividad física, alimentación y familia en edad escolar. Retos: nuevas tendencias en educación física, deporte y recreación, (34), 85-88.

Casimiro, A. \& Piéron, M. (2001). Incidencia de la práctica físico-deportiva de los padres hacia sus hijos durante la infancia y la adolescencia. Apunts. Educación Física y Deportes, 65, 100-104.

Cordente, C.A., García, P., \& Sillero, M. (2008). ¿Tienen los padres responsabilidad en la salud presente y futura de sus hijos adolescentes? Revista Internacional de derecho y gestión del deporte, (1), 36-48.

Cruz, J., Boixadós, M., Pintanel, M., Alonso, C., Mimbrero, J., \& Torregrosa, M. (1996). Identificación de conductas, actitudes y valores relacionados con el fairplay en deportistas jóvenes. Coleccioin ICD: Investigación en Ciencias del Deporte, (9).

Eccles, J. S. \& Harold, R. D. (1991). Gender differences in sport involvement: Applying the Eccles' expectancyvalue model. Journal of applied sport psychology, 3(1), 7-35. https://doi.org/10.1080/10413209108406432

Estrada, P. R., Vázquez, E. I. A., Gáleas, Á. M. V., Ortega, I. M. J., Serrano, M. D. L. P., \& Acosta, J. J. M. (2016). Beneficios psicológicos de la actividad física en el trabajo de un centro educativo. Retos: nuevas tendencias en educación física, deporte y recreación, (30), 203-206.FloresParedes, A., Vanegas-Pino., M., Y., \& Villamar-Barriga, M. Ó. (2020). Actividad física y su relación con el sobrepeso y obesidad en docentes de la Universidad Nacional del Altiplano Puno 2017. Sportis, 6(1), 61-79. https://doi.org/ 10.17979/sportis.2020.6.1.3448

Franco, E., Coterón, J., \& Gómez, V. (2017). Promoción de la actividad física en adolescentes: rol de la motivación y autoestima. PSIENCIA. Revista Latinoamericana de Ciencia Psicológica, 9(2), 1-15. doi: 10.5872/psiencia/ 9.2.24

Gálvez Casas, A., Rodríguez García, L., \& Velandrino Nicolás, A.P. (2009). La percepcioìn de competencia motriz y su repercusión en los niveles de práctica de actividad física escolar. Tandem: Didáctica de la educación física, (31), 62-70.

García Ferrando, M.(1997). Los españoles y el deporte, 19801995: Un estudio sociológico sobre comportamientos, actitudes y valores. Tirant lo Blanch, D.L.

González, J., Cayuela, D., \& López, C. (2019). Prosocialidad, educación física e inteligencia emocional en la escuela. Journal of Sport \& Health Research, 11(1), 17-32.

González, V. M., Gómez-López, M., \& Granero-Gallegos, A. (2019). Relación entre la satisfacción con las clases de Educación Física, su importancia y utilidad y la intención de práctica del alumnado de Educación Secundaria Obligatoria. Revista Complutense de Educación, 30(2), 479491.http://dx.doi.org/10.5209/RCED

Hair, J. F., Black, W. C., Babin, B., Anderson, R. E., \& Tatham, R. L. (2006). Multivariate data analysis. (6th ed.) New Jersey: Prentice Hall

Hohepa, M., Scragg, R., Schofield, G., Kolt, G. S., \& Schaaf, D. (2007). Social support for youth physical activity: Importance of siblings, parents, friends and school support across a segmented school day. International Journal of Behavioral Nutrition and Physical Activity, 4(54), 1-9. https://doi.org/10.1186/1479-5868-4-54

Jaramillo, M. M. C., Márquez, C. G., Jaime, M. D. L. M., Solís, L. O., Gómez, R. N. R., \& Hernández, L. V. (2017). Factores que determinan la práctica de actividad física en alumnos de secundaria. Revista Mexicana de Investigación en Cultura Física y Deporte, 5(7), 111-128.

Kotan, C'., Yaman, C'., \& Herguner, G. (2009). The effects of school and family factor son sports activities for sports students in primary schools: a case study of Sakarya. Beden Egitimi ve spor Bilimeri Dergisi, 3(1), 49-58.

Lizandra, J., \& Peiró-Velert, C. (2020). Las relaciones sociales y su papel en la motivación hacia la práctica de actividad física en adolescentes: Un enfoque cualitativo. Retos: Nuevas Tendencias en Educación Física, Deporte y Recreación, 37(37), 41-47.

López, A. T. (2019). Diferencias en los niveles de actividad física, grado de adherencia a la dieta mediterránea y autoconcepto físico en adolescentes en función del sexo. Retos, 36(36), 185-192.

McPherson, B., Curtis, J., \& Loy, J. (1989). The social significance of sport: an introduction to the sociology of sport. Champaign: Human Kinetics Books.

Mendoza, R., Batista, J. M., \& Saìgrera, M. R. (1994). Conductas de los escolares espanpoles relacionadas con la salud (1986-1990). Madrid: C.S.I.C., D.L.

Montil, M. (2004). Determinantes de la conducta de actividad física en población infantil. Tesis doctoral. Tecnología de los alimentos: E.T.S. de Ingenieros Agrónomos, Politécnica de Madrid.

Mota, J. \& Queiroìs, P. (1996). Children ìs behaviour. Physical Activity Regarding parent ìs perception vs children ìs activity. International Review for the Sociology of Sport, 31(2), 173-181. https://doi.org/10.1177/ 101269029603100204

Mota, J. \& Sallis, J. F. (2002). Actividade física e saúde: Factores de influência da actividade física nas crianças $e$ nos adolescentes. Oporto: campo de letras. 
Muñoz-Galiano, I., Hernández-García, R., \& Torres-Luque, G. (2019). Influencia del nivel educativo del progenitor sobre la práctica de actividad física en estudiantes de educación infantil. Journal of Sport and Health Research, 11(2), 161-170.

Nuviala, A. (2003). Las escuelas deportivas en un entorno rural aragonés. El caso del Servicio Comarcar Ribera Baja. Zaragoza: Gobierno de Aragón.

Ornelas, I. J., Perreira, K. M., \& Ayala, G. X. (2007). Parental influences on adolescent physical activity: a longitudinal study. International Journal of Behavioral Nutrition and Physical Activity, 4(1), 3. https://doi.org/10.1186/ 1479-5868-4-3

Pate, R.R., Freedson, P.S., Sallis, J.F., Taylos, W.C., Sirad, J., $\&$ Trost, S.G. (2002). Compliance with physical activity guidelines: Prevalence in a population of children and youth. Annals Epidemiology, 12(5), 303-308. https:// doi.org/10.1016/S1047-2797(01)00263-0

Pelegrín, A., García, H. G., \& De los Fayos, E. J. G. (2019). Estilos Educativos Parentales Percibidos en Adolescentes, Practicantes de Actividad Física, Federados y Competidores. Retos: Nuevas Tendencias en Educación Física, Deporte y Recreación, 36(36), 92-96.

Piéron, M., Ledent, M., Almond, L., Airstone, M., \& Newberry, I. (1996). Comparative analysis of youth lifestyle in selected European countries. Liège: University of Liège.

Quiroga, J. I. N., Ortega, F. Z., Granizo, I. R., Sánchez, A. L., Molero, P. P., \& Jiménez, J. L. U. (2019). Análisis de la relación entre los hábitos físico-saludables y la dieta con la obesidad en escolares de tercer ciclo de Primaria de la Provincia de Granada. Retos: Nuevas Tendencias en Educación Física, Deporte y Recreación, 35, 31-35.

Rodríguez, M.A. (2004). Un análisis de la educación familiar desde la teoría pedagógica: propuesta de bases fundamentales para un modelo integrado. (Tesis doctoral). Universidad de Valencia.

Ros-Fuentes, J.A. (2007). Actividad Física + Salud. Hacia un estilo de vida activo. Novograf.

Ruiz-Juan, F. (2000). Análisis diferencial de los comportamientos, motivaciones y demanda de actividades físico-deportivas del alumnado almeriense de Enseñanza Secundaria Postobligatoria y de la Universidad de Almeriìa. (Tesis doctoral). Universidad de Almería. Almería.

Sánchez, M.J. (1997). Personalidad y deporte. Ensayos Revista de Estudios de la Escuela Universitaria de Magisterio de Albacete, (12), 273-280.

Soubhi, H., Potvin, L., \& Paradis, G. (2004). Family process and parent's leisure time physical activity. American journal of health behavior, 28(3), 218-230. https://doi.org/ 10.5993/AJHB.28.3.3

Stucky-Ropp, R. C. \& DiLorenzo, T. M. (1993). Determinants of exercise in children. Preventive Medicine, 22(6), 880889.

Telama, R., Leskinen, E., \& Yang, X. (1996). Stability of habitual physical activity and sport participation: A longitudinal tracking study. Scandinavian Journal of Medicine \& Science in Sports, 6(6), 371-378. https:// doi.org/10.1111/j.1600-0838.1996.tb00109.x

Torre, E, (1998). La actividad físico deportiva extraescolar y su interrelacioìn con el área de Educación Física del alumnado de Enseñanzas Medias. (Tesis doctoral). Universidad de Granada. Granada.

Van der Horst, K., Paw, M.J., Twisk, J.W., \& Van Mechelen, W. (2007). A brief review on correlates of physical activity and sedentariness in Routh. Medicine and Science in Sports and Exercise, 39(8), 1241-1250. doi: 10.1249/ mss.0b013e318059bf35

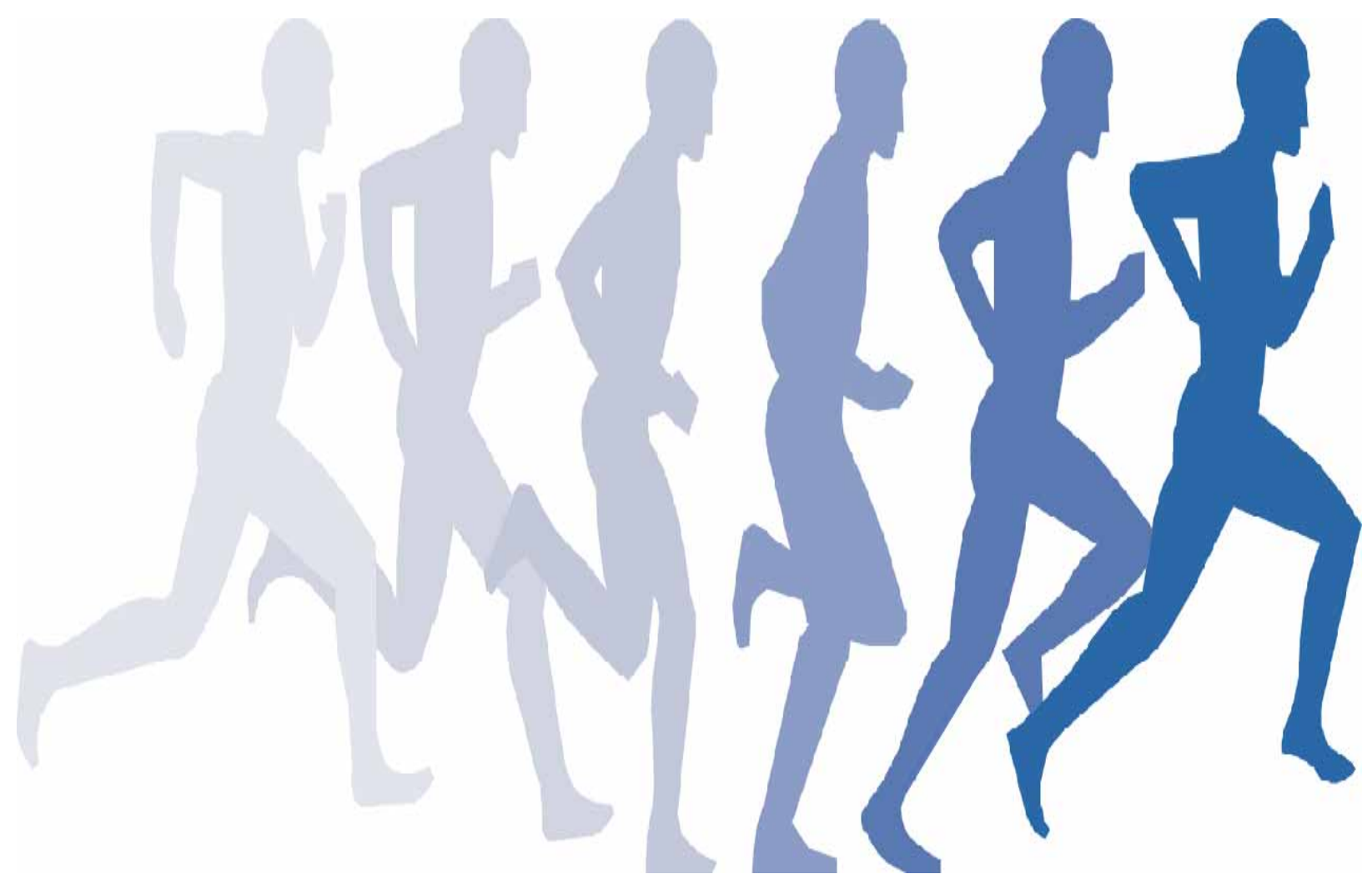

